\title{
Neurological Erectile Dysfunction
}

Secondary to Intradural Paraganglioma of the Cauda equina

\begin{tabular}{|l|l|l|}
\hline S.A. & Khan \\
\hline A.R. & & Iliya \\
\hline R.M. & & Washecka \\
\hline J.A. & & D'Agostino \\
\hline R.J. & & Wasnick \\
\hline
\end{tabular}

Departments of Urology and Neurosurgery, SUNY at Stony Brook, Stony Brook, N.Y., USA

\section{Key Words}

Erectile dysfunction

Impotence

Intradural paraganglioma

Cauda equina syndrome

Abstract

A 35-year-old white male presented with erectile dysfunction and areflexic bladder secondary to an intrathecal paraganglioma of the cauda equina. Erectile dysfunction has not been emphasized as a component of the cauda equina syndrome.

S.A. Khan, MD, Department of Urology, HSC - T9, SUNY at Stony Brook, Stony Brook, NY 11794 (USA)

\section{Introduction}

The cauda equina syndrome can result in complex damage to the sacral nerve roots with consequent sexual, bladder and bowel dysfunction. The syndrome is usually caused by benign degenerative disc disease or trauma and is rarely secondary to intraspinal tumors. We report a patient with erectile dysfunction and cauda equina syndrome due to an intrathecal paraganglioma at the mid-lumbar vertebral level. There have been several case reports of paraganglioma of the cauda equina [2].

$\Psi 9$

\section{Case Report}

This 35-year-old white male presented with progressive low back pain radiating into the lower extremities of 7 years duration. In addition, he developed paresthesias, loss of coordination, atrophy, and weakness of the lower extremities. For 2 years, the patient had loss of erections and also several episodes of urinary retention. He denied urinary incontinence, but he complained of intermittent episodes of fecal incontinence. Specifically, he could not distinguish flatus from feces.

Physical examination revealed diffuse gross atrophy, motor weakness, sensory loss, and absent reflexes corresponding to the lower lumbar and sacral dermatomes, worse on the left, in addition to diminished perineal sensation. The bulbocavernosus reflex was absent, and the anal sphincter was patulous. MRI scan of the lum-bosacral spine revealed an intradural mass lesion at the 
L3/L4 vertebral level occupying all of the spinal canal (fig. 1). Urodynamics showed a large capacity bladder, no detrusor contraction, absent sensation and large postvoid residual consistent with an areflexic bladder. The serum testosterone was $820 \mathrm{ng} / \mathrm{dl}$, prolactin was 5

$3 / 8 \mathrm{fc} \cdot 3 / 8 * \pi * \tau^{5} / 8$

I $\mid \mathrm{I}$

Fig. 1. MRI of lumbosacral spine demonstrating an intraspinal tumor (paraganglioma). $\mathrm{ng} / \mathrm{ml}$. A test dose of papaverine $(30 \mathrm{mg} / \mathrm{ml})$, injected intracorpo-really resulted in a rigid erection for $2 \mathrm{~h}$. Nocturnal rigiscan on three occasions documented lack of penile rigidity and tumescence.

A bilateral lumbar laminectomy with gross total excision of the large tumor was performed. The tumor was compressing the sacral nerve roots. The histology was consistent with a paraganglioma. There was no clinical evidence of catecholamine secretion.

120

Khan/Uiya/Washecka/D’Agostino/Wasnk :

Discussion

Neurologic impotence accounts for approximately $10-15 \%$ of patients with organic sexual dysfunction varying with patient population and referral sources [5]. Within this group infrequent and unusual diseases such as syringomyelia, transverse myelitis and spinal cord tumors must be considered as well as multiple sclerosis, diabetes mellitus, spinal cord trauma, lumbar disc disease and radical pelvic surgery. Although the differential can be esoteric, a thorough history and physical examination combined with MRI or CAT scan myelography of the spine are the best guides to establish the diagnosis. This is particularly true with the cauda equina syndrome.

The cauda equina syndrome is a constellation of complex symptoms due to compression of the nerve roots caudal to the conus medullaris which is usually below the first lumbar vertebra of the spinal column [6]. Significant parasympathetic nerve dysfunction of the second and third sacral segments may result in a combination of symptoms such as erectile failure, areflexic bladder and loss of epicritic discrimination between flatus and feces with resultant fecal incontinence [3]. Also, lower back pain with radiation into the lower extremities, motor weakness of the lower limbs, and reduced deep tendon reflexes are common [4]. Although bladder dysfunction has been described with the cauda equina syndrome [4], erectile dysfunction has not been emphasized adequately. Prognostically, nerve recovery or regeneration and clinical resolution of erectile dysfunction and bladde dysfunction may require several months to several year to resolve [1]. Consequently, the patient will requin long-term periodic evaluation and a rigiscan is a usefu tool to follow erectile function in these patients.

References

Hellstrom, P.; Kortelainen, P.; Kontturi, M: Late urodynamic findings after surgery for cauda equina syndrome caused by a prolapsed lumbar intervertebral disc. J. Urol. 135: 308 (1986). Ironside, J.W.: Paraganglioma of the cauda equina. J. Neurol. Neurosurg. Psychiat. 51: 740 (1988).

Last, R.J.: Anatomy regional and applied; 7th ed., p. 330 (Churchill-Livingstone, New York 1984).

Lipper, S.; Decker, R.E.: Paraganglioma of the cauda equina: A histologic, immunohistochemical, and ultrastructural study and review of the literature. Surg. Neurol. 22: 415 (1984). 
Padma-Nathan, H.; Goldstein, I.: Neurologic Assessment of the Impotent Patient; in Montague, Disorders of male sexual function, chap. 7, pp. 86-94 (Year Book Medical Publishers, Chicago 1988).

Rai, B.; Chopra, B.K.; Gupta, R.K.: Cauda equina syndrome. A clinical study. J. Indian med. Ass. 60: 47 (1973). 